\title{
Review Article \\ Recent Advances of Curcumin in the Prevention and Treatment of Renal Fibrosis
}

\author{
Xuejiao Sun, ${ }^{1}$ Yi Liu, ${ }^{1}$ Cheng Li, ${ }^{1}$ Xiting Wang, ${ }^{1}$ Ruyuan Zhu, ${ }^{1}$ Chenyue Liu, ${ }^{2}$ \\ Haixia Liu, ${ }^{1}$ Lili Wang, ${ }^{1}$ Rufeng $\mathrm{Ma},{ }^{1}$ Min Fu, ${ }^{3}$ Dongwei Zhang, ${ }^{4}$ and $\mathrm{Yu} \mathrm{Li}^{1}$ \\ ${ }^{1}$ Preclinical Medicine School, Beijing University of Chinese Medicine, Beijing 100029, China \\ ${ }^{2}$ Chinese Material Medical School, Beijing University of Chinese Medicine, Beijing 100029, China \\ ${ }^{3}$ The Research Institute of McGill University Health Center, Montreal, QC, Canada H4A 3J1 \\ ${ }^{4}$ Diabetes Research Center, Beijing University of Chinese Medicine, Beijing 100029, China \\ Correspondence should be addressed to Dongwei Zhang; dongwei1006@gmail.com and Yu Li; liyubeijing1973@163.com
}

Received 7 January 2017; Accepted 1 February 2017; Published 4 May 2017

Academic Editor: Ekaterina A. Ivanova

Copyright (c) 2017 Xuejiao Sun et al. This is an open access article distributed under the Creative Commons Attribution License, which permits unrestricted use, distribution, and reproduction in any medium, provided the original work is properly cited.

Curcumin, a polyphenol derived from the turmeric, has received attention as a potential treatment for renal fibrosis primarily because it is a relatively safe and inexpensive compound that contributes to kidney health. Here, we review the literatures on the applications of curcumin in resolving renal fibrosis in animal models and summarize the mechanisms of curcumin and its analogs (C66 and (1E,4E)-1,5-bis(2-bromophenyl) penta-1,4-dien-3-one(B06)) in preventing inflammatory molecules release and reducing the deposition of extracellular matrix at the priming and activation stage of renal fibrosis in animal models by consulting PubMed and Cnki databases over the past 15 years. Curcumin exerts antifibrotic effect through reducing inflammation related factors (MCP$1, \mathrm{NF}-\kappa \mathrm{B}$, TNF- $\alpha$, IL-1 $\beta$, COX-2, and cav-1) and inducing the expression of anti-inflammation factors (HO-1, M6PRBP1, and NEDD4) as well as targeting TGF- $\beta /$ Smads, MAPK/ERK, and PPAR- $\gamma$ pathways in animal models. As a food derived compound, curcumin is becoming a promising drug candidate for improving renal health.

\section{Introduction}

Curcumin, a polyphenol isolated from the Curcuma longa plant, is commonly known as turmeric in Asia (Figure 1) $[1,2]$. As a traditional used herbal medicine and also a food spice in global cuisines, turmeric was reported to have extensively clinical applications in various kinds of diseases, such as asthma, fibrosis, diabetes, and abdominal pain [24]. Curcumin is one of active ingredients in turmeric and has been reported to attenuate the expression of apoptotic and chemokine genes in rat model of unilateral ureteral obstruction (UUO) in 2000 [5]. After that, a lot of researchers conducted various experiments to study the effects and mechanisms regarding curcumin as a potential source in the prevention and treatment of renal fibrosis. In addition, there is no toxicity concern rising when curcumin is taken at the recommended doses, which increased the potential of therapeutic agent of this compound. A number of reviews concerning the use of Chinese medicine for fibrosis have been recently published $[4,6-8]$. This review intends to summarize the recent studies on curcumin in delaying advance of renal fibrosis through searching PubMed (https://www.pubmed.com/) and Cnki (https://www.cnki.com/) databases, which will provide additional evidence and also highlight the future research regarding curcumin in the management of kidney diseases.

Renal fibrosis is the principal process underlying the progression of chronic kidney disease (CKD) to end stage renal disease (ESRD). With a high prevalence of morbidity and mortality, CKD brings great pressure to patients and increases the burden on the society. In addition, currently there are no effective drugs to prevent the development of the ESRD. Characterized as glomerulosclerosis and tubular interstitial fibrosis, renal fibrosis is considered as a dynamic and converging process that consists of four overlapping phases: priming, activation, execution, and progression [9]. In the first stage, lasting inflammatory stimulation triggers 
<smiles>COc1ccc(/C=C/C(=O)CC(=O)/C=C/c2ccc(OC)c(O)c2)cc1O</smiles>

(a)<smiles>O=C1/C(=C/c2ccccc2C(F)(F)F)CCC/C1=C\C1CC=CC=C1C(F)(F)F</smiles>

(b)<smiles>O=C(/C=C/c1ccccc1Br)/C=C/c1ccccc1Br</smiles>

(c)

FIgURE 1: The chemical structure of curcumin (a), C66 (b), and B06 (c).

the activation of renal tubular epithelial cells and the infiltration of inflammatory cells, including lymphocytes, monocytes/macrophages, dendritic cells, and mast cells [10]. During the activation and execution stages, profibrotic cytokines are released from injured tubular cells accompanying the activation of matrix-producing cells. It is accepted that the myofibroblasts are the main source of extracellular matrix (ECM), which derived from renal interstitial fibroblasts, bone marrow-derived fibrocytes, vascular pericytes, and endothelial and tubular cells by epithelial-to-mesenchymal transdifferentiation (EMT) [11]. The excessive deposition of ECM such as fibronectin and types I and III collagen contributes to the development of renal fibrosis. In the final stage, the renal structure and function gradually disappear with sustaining ECM deposition, which leads to the undesirable consequence of fibrosis.

\section{Curcumin Is Involved in the Priming Stage of Renal Fibrosis}

At the priming stage of renal fibrosis, inflammation initiates a fibrotic process [12]. Sustaining inflammatory stimulus triggers the activation of renal tubular epithelial cells and the infiltration of inflammatory cells, including lymphocytes, monocytes/macrophages, dendritic cells, and mast cells. Curcumin has been demonstrated to regulate multiple proinflammatory molecules and reduce recruitment of inflammatory macrophages [13-16] in various animal renal fibrosis models (Table 1).

Monocyte chemotactic protein-1 (MCP-1) is an important medium for monocyte/macrophage infiltration and a principle cytokine that may induce tubulointerstitial fibrosis (TIF) [17]. Macrophages are attracted to the site of injury by MCP-1 and its receptor CCR2. Blocking MCP-1/CCR2 pathway was shown to prevent kidney fibrosis through reducing recruitment of M1 inflammatory macrophages [18]. In the UUO rats models [5], curcumin treatment $(0.5 \mathrm{~mL}$ of $30 \mathrm{mg} / \mathrm{mL}$ for 10 days, subcutaneous injection) significantly attenuated MCP-1mRNA overexpression in the obstructed kidney compared with that of control [5]. Further, curcumin treatment also decreased MCP-1 level in the factor-H-deficient mice
(30 mg/kg for 5 weeks, intraperitoneal injection) [19] and lipopolysaccharide (LPS) stimulated mice $(5 \mathrm{mg} / \mathrm{kg}$ for 3 days, intraperitoneal injection) [20].

Under the stimulation of inflammatory factors (interleukin (IL), tumor necrosis factor $\alpha$ (TNF- $\alpha)$ ), nuclear factor-kappa $\mathrm{B}(\mathrm{NF}-\kappa \mathrm{B})$ is activated and this activation further promotes the expression of transforming growth factor $\beta$ (TGF- $\beta$ ) 1, intercellular adhesion molecule-1, and other fibrogenesis factors [33-35]. It has been demonstrated that curcumin treatment $(100 \mathrm{mg} / \mathrm{kg} /$ day for 8 weeks, oral gavage) suppressed NF- $\kappa \mathrm{B}$ activation, prevented inhibitor of NF- $\kappa \mathrm{B}(\mathrm{I} \kappa \mathrm{Ba})$ degradation, and decreased intercellular adhesion molecule-1 protein expression in streptozotocin(STZ-) induced diabetic nephropathy rats [15], which was also reflected in LPS-induced kidney inflammation mice [20].

Proinflammatory cytokines, including TNF- $\alpha$ and IL-1, are involved in the development of chronic kidney disorders, including glomerulonephritis [36]. In 5/6 nephrectomy $(5 / 6 \mathrm{Nx})$ rats, the high levels of TNF- $\alpha$ and IL- $1 \beta$ further triggered the production of cytosolic phospholipase A2 (cPLA2), calcium-independent intracellular PLA2 (iPLA2), and cyclooxygenase (COX) isoforms, which might contribute to inflammation [9]. Curcumin treatment $(75 \mathrm{mg} / \mathrm{kg}$, oral gavage) for 10 weeks significantly reduced the levels of the above-mentioned factors in 5/6 Nx rats [23]. In addition, administration of turmeric-based diet (5\% w/w for 30 days) significantly decreased TNF- $\alpha$ mRNA expression in UUO rats [26]. C66 (0.2 mg/kg for 6 weeks, oral gavage), a novel curcumin derivative, has also been reported to reduce the production of TNF- $\alpha$, IL- $1 \beta, \mathrm{COX}-2$, and NF- $\kappa \mathrm{B}$ in high glucose stimulated diabetic rats [37]. The above-mentioned results suggest that curcumin and its analogs may have strong ability of anti-inflammation in different renal rodent's diseases models.

Heme oxygenase-1 (HO-1) is the inducible isoform of the rate-limiting enzyme involved in the degradation of heme. It is a cytoprotective molecule that could restore renal function via resolving fibrosis factors [38, 39]. In anti-Thyl glomerulonephritis rats, curcumin treatment (10 to $200 \mathrm{mg} / \mathrm{kg}$, intraperitoneal injection) dose-dependently induced the expression of $\mathrm{HO}-1$ in glomerular cells and 


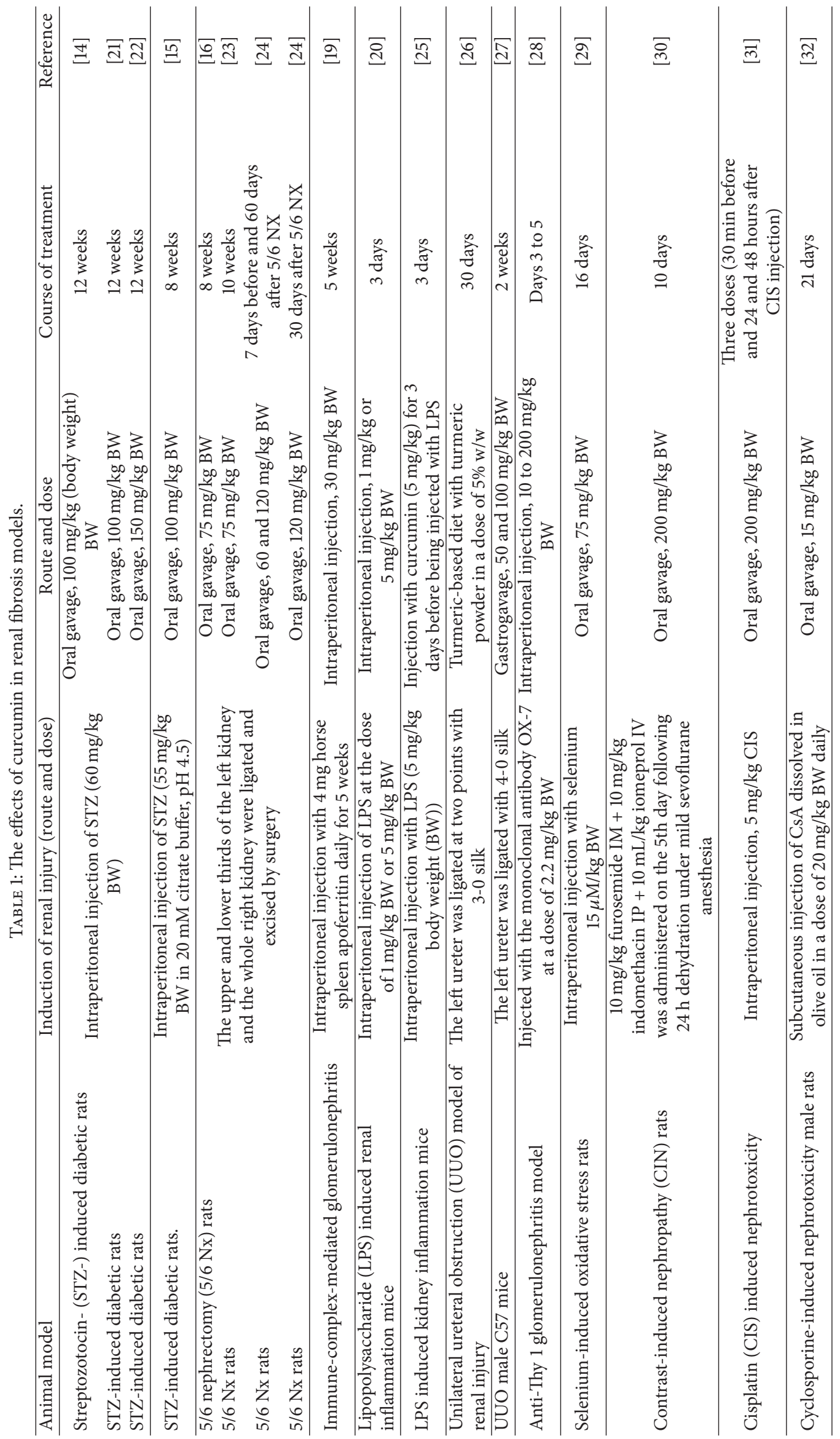


antithymocyte serum nephritic rats [28]. The association of curcumin with HO-1 was further demonstrated by using zinc protoporphyrin (HO-1 inhibitor) in anti-Thyl glomerulonephritis rats which resulted in loss of beneficial effects of curcumin on fibrosis and proteinuria [28]. In addition, curcumin treatment also increased $\mathrm{HO}-1$ expression in the kidney of UUO rats [5]. The results also indicate that HO1 agonists may offer new opportunity for renal diseases treatment.

Neural precursor cell expressed, developmentally downregulated 4 (NEDD4) family is closely related to inflammation, and mice lacking Nedd4 family interacting protein1 developed severe inflammation in the skin and lung [40]. Mannose-6-phosphate receptor binding protein 1 (M6PRBP1) is involved in the metabolism of intracellular lipid. The levels of M6PRBP-1 and NEDD4 were reduced in response to LPS insulation [25]. Curcumin treatment ( $5 \mathrm{mg} / \mathrm{kg}$ for 3 days, intraperitoneal injection) increased renal M6PRBP1 and NEDD4 expression in LPS-induced kidney inflammation in Kunming mice [19]. Furthermore, the investigators also found that curcumin could inhibit the activation of mitogen-activated protein kinases (MAPK) and JNKp38MAPK pathways by gene chip analysis [25], suggesting an important role in inflammation response [41, 42]. The results suggest that curcumin may have the effects on inflammatory cells proliferation, differentiation, and migration.

Caveolin-1 (cav-1) activation modulates innate immunity, inflammation, vascular permeability, and leukocyte migration [43]. Cav-1 binds to toll-like receptor 4 (TLR4), then mediates NF- $\kappa \mathrm{B}$ activation, and triggers the inflammatory response [44]. Curcumin treatment reduced cav-1 phosphorylation at Tyr14 and TLR4 activation in STZ-induced diabetic rats $(100 \mathrm{mg} / \mathrm{kg}$ for 12 weeks, oral gavage) and high glucose stimulated mouse podocyte cell (curcumin, 1 to $10 \mu \mathrm{M})$ [14].

In short, curcumin has been demonstrated to exhibit anti-inflammation properties in different kidney diseases models by reducing inflammatory molecules release (MCP$1, \mathrm{NF}-\kappa \mathrm{B}, \mathrm{TNF}-\alpha$, IL- $1 \beta, \mathrm{COX}-2$, and cav- 1 ) and inducing the expression of anti-inflammation factors (HO-1, M6PRBP1, and NEDD4), suggesting that it could play a contributing role in preventing the initiation of renal fibrosis.

\section{Curcumin Is Also Actively Involved in Activation Stage of Renal Fibrosis}

At the activation stage, profibrotic cytokines and factors are released from injured tubular cells, which stimulate the myofibroblasts to produce ECM. In addition, EMT further contributes to transdifferentiating endothelial and tubular cells to myofibroblasts [45]. An experiment performed by Sun et al. demonstrated that administration of curcumin $(100 \mathrm{mg} / \mathrm{kg}$ for 12 weeks, oral gavage) prevented EMT through increasing the expression of epithelia cadherin, synaptopodin, and reducing expression of mesenchymal asmooth muscle actin ( $\alpha$-SMA), fibroblast-specific protein 1 in the diabetic rats [21]. The possible mechanisms underlying these effects might be involved in suppressing the phosphorylation of cav-1 at Tyr14 and increasing stabilization of cav-1 and $\beta$-catenin. In addition, $\beta$-catenin favors EMT and renal fibrosis [46-48]. Curcumin inhibited high glucose induced dissociation of $\beta$-catenin from cav-1 and decreased active $\beta$-catenin expression [21]. In our group we also found that curcumin could inhibit the occurrence of EMT in renal tubular epithelial cells via regulating several sites of the TGF$\beta /$ Smads signal transduction pathway in UUO rats [49]. The inhibitory effect of curcumin on EMT was also demonstrated in cisplatin-induced renal fibrosis rats [31].

In addition, curcumin was proved to ameliorate EMT in TGF- $\beta 1$ stimulated proximal tubular HK-2 cells through ERK and PPAR- $\gamma$ dependent pathway [50]. Curcumin also exhibited similar effect in high glucose exposed NRK-52E kidney tubular epithelial cell through stimulating nuclear factor(erythroid-derived 2-) like 2- (Nrf2-) mediated upregulation of HO-1 [51].

The disequilibrium between oxidant and antioxidant system contributes to development of renal damage [52]. Curcumin $(75 \mathrm{mg} / \mathrm{kg}$ for 16 days; oral gavage) was evidenced to inhibit the increase of inducible nitric oxide synthase (iNOS) expression in kidney in selenium-induced toxicity in Wistar rats [29]. The reducing level of iNOS facilitated removing oxidative/nitrosative stress. The protective effect of curcumin was more obvious in pretreatment group (administration of curcumin before selenium, $24 \mathrm{~h}$ ) than simultaneous or posttreatment group (administration of curcumin after selenium treatment, $24 \mathrm{~h}$ ). In rats with contrast-induced nephropathy [30], 5/6 Nx [24], and cisplatin-induced nephrotoxicity [31], curcumin treatment $(60$ and $120 \mathrm{mg} / \mathrm{kg}$ for 60 days [24]) increased antioxidant profiles (e.g., superoxide dismutase (SOD), enzymes catalase, glutathione reductase, glutathione peroxidase, and glutathione) and decreased oxidant profile (malondialdehyde) in the kidneys. Further, curcumin may exhibit renoprotective effect through Nrf2 translocation [31, 53], which beneficially contributes to ameliorating cisplatin-induced loss of tight junction proteins (claudin-2 and occludin) and adherens junction protein (E-cadherin and $\beta$-catenin) [31]. It is demonstrated that Nrf2 exerted cytoprotective effect through binding to antioxidant response elements $[54,55]$ (Figure 2). Interestingly, curcumin also exerted similar protective effects against oxidant stress induced renal damage between the pretreatment and posttreatment groups in $5 / 6 \mathrm{Nx}$ rats. The inconsistency of the effects of curcumin administration approach between $5 / 6 \mathrm{Nx}$ and seleniuminduced rats may be owing to different renal diseases models, duration, and dosage. In rats with cyclosporineinduced nephrotoxicity, curcumin treatment $(15 \mathrm{mg} / \mathrm{kg}$ for 21 days, subcutaneous injection) ameliorate renal injury by decreasing glutathione S-transferase immunoreactivity [32] which indicated that exogenous antioxidant curcumin might compensate the need of the renal cells to the endogenous glutathione antioxidant [42].

TGF- $\beta /$ Smads signaling is considered as the most important pathway in the development of renal fibrosis [45]. TGF$\beta 1$ regulates the synthesis and degradation of ECM and induces the activities of fibrogenic cytokines that contributed to the development of fibrosis. TGF- $\beta 1$ overexpression heavily favors fibrotic kidney disease [56]. Curcumin treatment inhibited TGF- $\beta 1 \mathrm{mRNA}$ expression [19]. And this inhibitory 


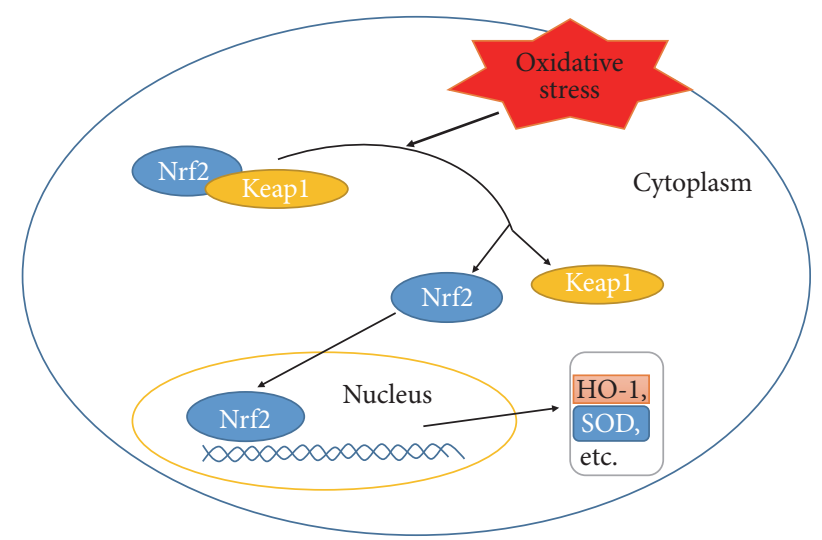

Figure 2: Nrf2 signaling pathway. Nrf2, as a transcription factor, resides within cytoplasm binding to the actin-associated Keap1 protein and is normally degraded. Upon oxidation stress, the association will be disrupted, resulting in the translocation of Nrf2 to nuclei and then increased expression of cytoprotective enzymes (HO-1, SOD, etc.).

effect was mediated through reducing the phosphorylation of Smad2 and Smad3 [27]. In addition, pretreatment of curcumin resisted renal fibrosis by downregulating TGF$\beta 1$ receptor II in TGF- $\beta 1$ stimulated NRK49F rat renal fibroblasts [57]. B06, one of the curcumin analogs, has also been proved to reduce the expression of collagen IV and fibronectin which further favored attenuating the accumulation of extracellular matrix and glomerular mesangial proliferation [58].

Furthermore, sphingosine 1-phosphate (S1P) activates TGF- $\beta$ and contributed the renal fibrosis process [59]. However, the formation of S1P is catalyzed by sphingosine kinase 1 (SphK1) [60]. Huang et al. found that curcumin treatment ( $150 \mathrm{mg} / \mathrm{kg}$ for 12 weeks, oral gavage) significantly inhibited expression and activity of SphK1 and the production of S1P in STZ-induced diabetic rats [22].

In addition, our group found that curcumin treatment $(20 \mu \mathrm{M}$ for $72 \mathrm{~h})$ significantly decreased the expression of collagen I, $\alpha$-SMA, and chemokine receptor 7 (CCR7), as well as TGF- $\beta 1$ secretion in human circulating fibrocytes [61]. The inhibitory effect of curcumin on the differentiation and migration of human circulating fibrocytes is likely through regulating the CCR7/CCL21 signaling pathway, in particular by reducing CCR7 expression.

The MAPK/ERK signaling pathway is also involved in the development of renal fibrosis [62,63]. Pretreatment with curcumin blocked angiotensin II- (Ang-II-) induced profibrotic responses in renal tubular epithelial cells [64]. Ang-II exerted its fibrotic response and hypertension effect through TGF $\beta 1$ MAPK/ERK pathway [65] and renin-angiotensin system. Pan et al. [37] further demonstrated that C66 prevented STZinduced diabetic nephropathy through inhibition of MAPK mediated angiotensin converting enzyme (ACE) expression. Wang et al. [66] also found that the antifibrotic effect of C66 was exhibited through inhibition of JNK phosphorylation and p300/CBP-mediated histone acetylation. It is demonstrated that inhibition of histone deacetylase prevented renal interstitial fibroblasts activation and renal tubular cell apoptosis in a rat renal interstitial fibroblast line (NRK-49F) and in UUO mice models [67].

Emerging evidence suggests that peroxisome proliferatoractivated receptor- $\gamma$ (PPAR- $\gamma$ ) is implicated in cell cycle [68] and its agonists exert protective effect on glucose control, alleviating proteinuria and inhibiting tissue fibrosis [69]. In UUO mice, curcumin treatment $(50 \mathrm{mg} / \mathrm{kg}$ and $100 \mathrm{mg} / \mathrm{kg}$ for 14 days, oral gavage) increased PPAR- $\gamma$ expression and decreased phosphorylated Smad 2/3 [27]. This was also reflected in TGF- $\beta 1$ stimulated proximal tubular epithelial cell HK-2 cells [50] and 5/6 Nx rats [16]. Since PPAR- $\gamma$ is also associated with ACE [70, 71], it is also useful to probe the effect of curcumin on the ACE expression in renal diseases.

In summary, at activation stage of renal fibrosis, curcumin treatment inhibits EMT and rebuilds the oxidativeantioxidant balance. In addition, curcumin shows antifibrogenic properties by regulating TGF- $\beta$ expression and blocking MAPK/ERK and PPAR- $\gamma$ pathways.

\section{Outlook and Conclusions}

Curcumin has been demonstrated to be beneficially involved in resolving renal fibrosis at priming and activation stages through preventing inflammation initiation, rebuilding redox balance, inhibiting EMT, and resolving ECM excess deposition. These actions are mediated by reducing inflammation related factors (MCP-1, NF- $\kappa$ B, TNF- $\alpha$, IL- $1 \beta$, COX-2, and cav-1) and inducing the expression of anti-inflammation factors (HO-1, M6PRBP1, and NEDD4) as well as targeting TGF- $\beta /$ smads, MAPK/ERK, and PPAR- $\gamma$ pathways in animal models (Figure 3 ). In addition, no data supports the notion that curcumin could restore renal injury during ESRD so far. Meanwhile, cautions must be excised that pretreatment and posttreatment may affect the effects of curcumin on renal fibrosis. Prospective studies are also needed to further elucidate the effects of curcumin in the development of renal fibrosis with in-depth understanding of this disease.

However, concerns are rising regarding the efficacy of curcumin in the management of renal fibrosis owing to its inherent low bioavailability. In most of the studies, curcumin was administrated by oral gavage. Further investigations are needed to explore the real active ingredients of curcumin after administration. Fortunately, some of curcumin derivatives with good bioavailability (such as C66 and B06) and new formulations of curcumin have been developed in recently years. However, the efficacy and safety of these new analogs and formulations remain largely unexplored. Taken together, as a food derived compound with golden-yellow fluorescence, curcumin may offer a new option in the treatment of renal fibrosis and also provide a new druggable chemical structure for chemists in designing new antifibrosis drug candidates. 


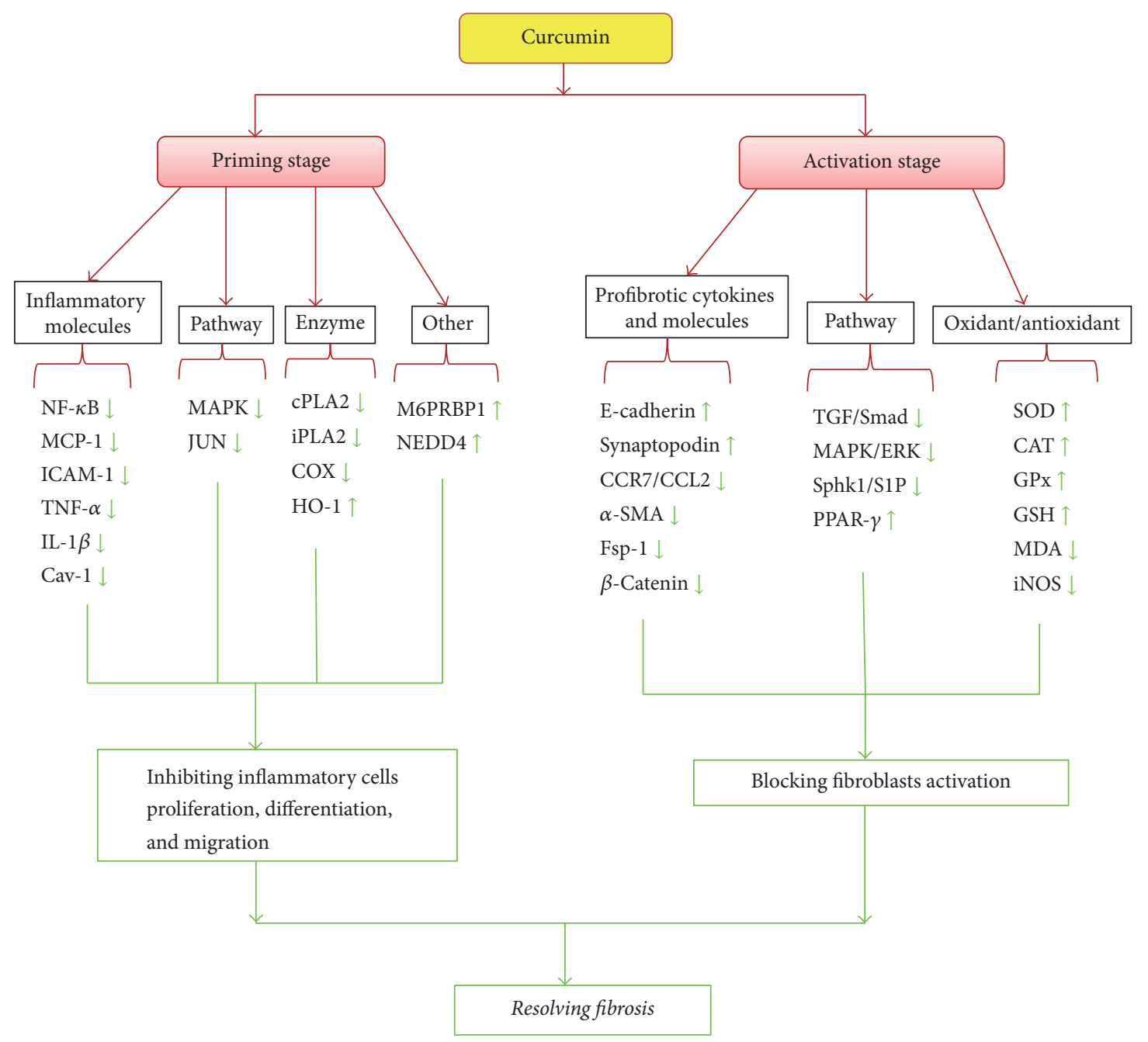

FIGURE 3: Curcumin plays a protective role at the priming and the activation stage of renal fibrosis. At the priming stage, curcumin reduces proinflammatory molecular activity and blocks inflammation associated signaling pathways. At the activation stage, curcumin inhibits the expression of renal fibrosis markers, rebuilds the redox balance, blocks MAPK/ERK pathway and TGF- $\beta$ /Smads pathway, and increases PPAR$\gamma$ expression. NF- $\kappa$ B, nuclear factor-kappa B; MCP-1, monocyte chemotactic protein 1; ICAM-1, intercellular adhesion molecule 1; TNF- $\alpha$, tumor necrosis factor $\alpha$; IL-1 $\beta$, interleukin-1 $\beta$; Cav-1, Caveolin-1; MAPK, mitogen-activated protein kinase; cPLA2, cytosolic phospholipase A2; iPLA2, calcium-independent intracellular PLA2; COX, cyclooxygenase; HO-1, heme oxygenase-1; CCR7, chemokine receptor 7; CCL21, chemokine ligand 21; $\alpha$-SMA, $\alpha$ smooth muscle actin; Fsp-1, fibroblast-specific protein 1; TGF, transforming growth factor; Sphk1, sphingosine kinase 1; S1P, sphingosine 1-phosphate; PPAR- $\gamma$, peroxisome proliferators-activated receptor- $\gamma$; SOD, superoxide dismutase; CAT, catalase; GR, glutathione reductase; GPx, glutathione peroxidase; GSH, glutathione; MDA, malondialdehyde; iNOS, inducible nitric oxide synthase; NEDD4, neural precursor cell expressed, developmentally downregulated 4; M6PRBP1, mannose-6-phosphate receptor binding protein 1.

\section{Conflicts of Interest}

The authors declare that they have no conflicts of interest.

\section{Acknowledgments}

This work was supported by grants from National Natural Science Foundation of China (nos. 81173642, 81573716, and 81273995) and the 111 Project of MOE (B07007).

\section{References}

[1] S. Kössler, C. Nofziger, M. Jakab, S. Dossena, and M. Paulmichl, "Curcumin affects cell survival and cell volume regulation in human renal and intestinal cells," Toxicology, vol. 292, no. 2-3, pp. 123-135, 2012.

[2] D.-w. Zhang, M. Fu, S.-H. Gao, and J.-L. Liu, "Curcumin and diabetes: a systematic review," Evidence-Based Complementary and Alternative Medicine, vol. 2013, Article ID 636053, 16 pages, 2013.

[3] S. Prasad and B. Aggarwal, "Turmeric, the golden spice: from traditional medicine to modern medicine," in Herbal Medicine: Biomolecular and Clinical Aspects, I. F. F. Benzie and S. WachtelGalor, Eds., pp. 263-288, CRC Press, Boca Raton, Fla, USA, 2011.

[4] D. Lelli, A. Sahebkar, T. P. Johnston, and C. Pedone, "Curcumin use in pulmonary diseases: state of the art and future perspectives," Pharmacological Research, vol. 115, pp. 133-148, 2017. 
[5] E. A. Jones, A. Shahed, and D. A. Shoskes, "Modulation of apoptotic and inflammatory genes by bioflavonoids and angiotensin II inhibition in ureteral obstruction," Urology, vol. 56, no. 2, pp. 346-351, 2000.

[6] J. Trujillo, Y. I. Chirino, E. Molina-Jijón, A. C. AndéricaRomero, E. Tapia, and J. Pedraza-Chaverrí, "Renoprotective effect of the antioxidant curcumin: recent findings," Redox Biology, vol. 1, no. 1, pp. 448-456, 2013.

[7] L.-C. Li and L.-D. Kan, "Traditional Chinese medicine for pulmonary fibrosis therapy: progress and future prospects," Journal of Ethnopharmacology, vol. 198, pp. 45-63, 2017.

[8] J. Xia, L.-Q. He, and X. Su, "Interventional mechanisms of herbs or herbal extracts on renal interstitial fibrosis," Journal of Integrative Medicine, vol. 14, no. 3, pp. 165-173, 2016.

[9] Y. Liu, "Cellular and molecular mechanisms of renal fibrosis," Nature Reviews Nephrology, vol. 7, no. 12, pp. 684-696, 2011.

[10] X.-M. Meng, D. J. Nikolic-Paterson, and H. Y. Lan, "Inflammatory processes in renal fibrosis," Nature Reviews Nephrology, vol. 10, no. 9, pp. 493-503, 2014.

[11] B. Conway and J. Hughes, "Cellular orchestrators of renal fibrosis," QJM, vol. 105, no. 7, pp. 611-615, 2012.

[12] D. J. Nikolic-Paterson, S. Wang, and H. Y. Lan, "Macrophages promote renal fibrosis through direct and indirect mechanisms," Kidney International Supplements, vol. 4, no. 1, pp. 3438, 2014.

[13] N. Kuwabara, S. Tamada, T. Iwai et al., "Attenuation of renal fibrosis by curcumin in rat obstructive nephropathy," Urology, vol. 67, no. 2, pp. 440-446, 2006.

[14] L.-N. Sun, Z.-Y. Yang, S.-S. Lv, X.-C. Liu, G.-J. Guan, and G. Liu, "Curcumin prevents diabetic nephropathy against inflammatory response via reversing caveolin-1 Tyr14 phosphorylation influenced TLR4 activation," International Immunopharmacology, vol. 23, no. 1, pp. 236-246, 2014.

[15] V. Soetikno, F. R. Sari, P. T. Veeraveedu et al., "Curcumin ameliorates macrophage infiltration by inhibiting NF- $\kappa$ B activation and proinflammatory cytokines in streptozotocin induceddiabetic nephropathy," Nutrition and Metabolism, vol. 8, no. 1, article 35, 2011.

[16] S. S. Ghosh, H. D. Massey, R. Krieg et al., "Curcumin ameliorates renal failure in 5/6 nephrectomized rats: role of inflammation," American Journal of Physiology-Renal Physiology, vol. 296, no. 5, pp. F1146-F1157, 2009.

[17] T. Wada, K. Furuichi, N. Sakai et al., "Gene therapy via blockade of monocyte chemoattractant protein-1 for renal fibrosis," Journal of the American Society of Nephrology, vol. 15, no. 4, pp. 940-948, 2004.

[18] K. Kitagawa, T. Wada, K. Furuichi et al., "Blockade of CCR2 ameliorates progressive fibrosis in kidney," The American Journal of Pathology, vol. 165, no. 1, pp. 237-246, 2004.

[19] A. Jacob, L. Chaves, M. T. Eadon, A. Chang, R. J. Quigg, and J. J. Alexander, "Curcumin alleviates immune-complex-mediated glomerulonephritis in factor-H-deficient mice," Immunology, vol. 139, no. 3, pp. 328-337, 2013.

[20] F. Zhong, H. Chen, L. Han, Y. Jin, and W. Wang, "Curcumin attenuates lipopolysaccharide-induced renal inflammation," Biological and Pharmaceutical Bulletin, vol. 34, no. 2, pp. 226-232, 2011.

[21] L.-N. Sun, Z.-X. Chen, X.-C. Liu, H.-Y. Liu, G.-J. Guan, and G. Liu, "Curcumin ameliorates epithelial-to-mesenchymal transition of podocytes in vivo and in vitro via regulating caveolin-1," Biomedicine and Pharmacotherapy, vol. 68, no. 8, pp. 1079-1088, 2014.
[22] J. Huang, K. Huang, T. Lan et al., "Curcumin ameliorates diabetic nephropathy by inhibiting the activation of the SphK1-S1P signaling pathway," Molecular and Cellular Endocrinology, vol. 365, no. 2, pp. 231-240, 2013.

[23] S. S. Ghosh, R. Krieg, H. D. Massey et al., "Curcumin and enalapril ameliorate renal failure by antagonizing inflammation in 5/6 nephrectomized rats: role of phospholipase and cyclooxygenase," American Journal of Physiology - Renal Physiology, vol. 302, no. 4, pp. F439-F454, 2012.

[24] E. Tapia, Z. L. Zatarain-Barrón, R. Hernández-Pando et al., "Curcumin reverses glomerular hemodynamic alterations and oxidant stress in 5/6 nephrectomized rats," Phytomedicine, vol. 20, no. 3-4, pp. 359-366, 2013.

[25] F. Zhong, H. Chen, Y. Jin, S. Guo, W. Wang, and N. Chen, "Analysis of the gene expression profile of curcumin-treated kidney on endotoxin-induced renal inflammation," Inflammation, vol. 36, no. 1, pp. 80-93, 2013.

[26] R. M. Hashem, H. M. Soliman, and S. F. Shaapan, "Turmericbased diet can delay apoptosis without modulating NF- $\kappa \mathrm{B}$ in unilateral ureteral obstruction in rats," Journal of Pharmacy and Pharmacology, vol. 60, no. 1, pp. 83-89, 2008.

[27] X. Zhou, J. Zhang, C. Xu, and W. Wang, "Curcumin ameliorates renal fibrosis by inhibiting local fibroblast proliferation and extracellular matrix deposition," Journal of Pharmacological Sciences, vol. 126, no. 4, pp. 344-350, 2014.

[28] J. Gaedeke, N. A. Noble, and W. A. Border, "Curcumin blocks fibrosis in anti-Thy 1 glomerulonephritis through up-regulation of heme oxygenase 1," Kidney International, vol. 68, no. 5, pp. 2042-2049, 2005.

[29] R. Manikandan, R. Thiagarajan, S. Beulaja, G. Sudhandiran, and M. Arumugam, "Curcumin protects against hepatic and renal injuries mediated by inducible nitric oxide synthase during selenium-induced toxicity in Wistar rats," Microscopy Research and Technique, vol. 73, no. 6, pp. 631-637, 2010.

[30] M. Buyuklu, F. Mehmet Kandemir, M. Ozkaraca, T. Set, E. Murat Bakirci, and E. Topal, "Protective effect of curcumin against contrast induced nephropathy in rat kidney: what is happening to oxidative stress, inflammation, autophagy and apoptosis?" European Review for Medical and Pharmacological Sciences, vol. 18, no. 4, pp. 461-470, 2014.

[31] J. Trujillo, E. Molina-Jijón, O. N. Medina-Campos et al., "Curcumin prevents cisplatin-induced decrease in the tight and adherens junctions: relation to oxidative stress," Food and Function, vol. 7, no. 1, pp. 279-293, 2016.

[32] E. A. Abdel Fattah, H. E. Hashem, F. A. Ahmed, M. A. Ghallab, I. Varga, and S. Polak, "Prophylactic role of curcumin against cyclosporine-induced nephrotoxicity: histological and immunohistological study," General Physiology and Biophysics, vol. 29, no. 1, pp. 85-94, 2010.

[33] H.-Y. Xue, L. Yuan, Y.-J. Cao, Y.-P. Fan, X.-L. Chen, and X.-Z. Huang, "Resveratrol ameliorates renal injury in spontaneously hypertensive rats by inhibiting renal micro-inflammation," Bioscience Reports, vol. 36, no. 3, Article ID e00339, 2016.

[34] T. D. Gilmore, "Introduction to NF- $\kappa$ B: players, pathways, perspectives," Oncogene, vol. 25, no. 51, pp. 6680-6684, 2006.

[35] R. P. Nagarajan, F. Chen, W. Li et al., "Repression of transforming-growth-factor- $\beta$-mediated transcription by nuclear factor $\kappa \mathrm{B}$," Biochemical Journal, vol. 348, no. 3, pp. 591-596, 2000.

[36] F. Kayama, T. Yoshida, Y. Kodama, T. Matsui, J. M. Matheson, and M. I. Luster, "Pro-inflammatory cytokines and interleukin 
6 in the renal response to bacterial endotoxin," Cytokine, vol. 9, no. 9, pp. 688-695, 1997.

[37] Y. Pan, Y. Wang, L. Cai et al., "Inhibition of high glucoseinduced inflammatory response and macrophage infiltration by a novel curcumin derivative prevents renal injury in diabetic rats," British Journal of Pharmacology, vol. 166, no. 3, pp. 11691182, 2012.

[38] M. Correa-Costa, P. Semedo, A. P. F. S. Monteiro et al., "Induction of heme oxygenase-1 can halt and even reverse renal tubule-interstitial fibrosis," PLoS ONE, vol. 5, no. 12, Article ID e14298, 2010.

[39] X. Chen, S.-Y. Wei, J.-S. Li et al., "Overexpression of heme oxygenase-1 prevents renal interstitial inflammation and fibrosis induced by unilateral ureter obstruction," PLoS ONE, vol. 11, no. 1, Article ID e0147084, 2016.

[40] P. M. Oliver, X. Cao, G. S. Worthen et al., "Ndfip1 protein promotes the function of itch ubiquitin ligase to prevent $\mathrm{T}$ cell activation and T helper 2 cell-mediated inflammation," Immunity, vol. 25, no. 6, pp. 929-940, 2006.

[41] X. Che, Q. Wang, Y. Xie et al., "Astragaloside IV suppresses transforming growth factor- $\beta 1$ induced fibrosis of cultured mouse renal fibroblasts via inhibition of the MAPK and NF$\kappa \mathrm{B}$ signaling pathways," Biochemical and Biophysical Research Communications, vol. 464, no. 4, pp. 1260-1266, 2015.

[42] W. Wang, P.-H. Zhou, C.-G. Xu, X.-J. Zhou, W. Hu, and J. Zhang, "Baicalein attenuates renal fibrosis by inhibiting inflammation via down-regulating NF- $\kappa \mathrm{B}$ and MAPK signal pathways," Journal of Molecular Histology, vol. 46, no. 3, pp. 283-290, 2015.

[43] S. Akira, K. Takeda, and T. Kaisho, "Toll-like receptors: critical proteins linking innate and acquired immunity," Nature Immunology, vol. 2, no. 8, pp. 675-680, 2001.

[44] G. H. Tesch, "Macrophages and diabetic nephropathy," Seminars in Nephrology, vol. 30, no. 3, pp. 290-301, 2010.

[45] Y. B. Y. Sun, X. Qu, G. Caruana, and J. Li, "The origin of renal fibroblasts/myofibroblasts and the signals that trigger fibrosis," Differentiation, vol. 92, no. 3, pp. 102-107, 2016.

[46] S. Hao, W. He, Y. Li et al., "Targeted inhibition of $\beta$-catenin/CBP signaling ameliorates renal interstitial fibrosis," Journal of the American Society of Nephrology, vol. 22, no. 9, pp. 1642-1653, 2011.

[47] J. Heuberger and W. Birchmeier, "Interplay of cadherinmediated cell adhesion and canonical Wnt signaling," Cold Spring Harbor Perspectives in Biology, vol. 2, no. 2, Article ID a002915, 2010.

[48] F. Galbiati, D. Volonte, A. M. C. Brown et al., "Caveolin-1 expression inhibits Wnt/ $\beta$-catenin/Lef-1 signaling by recruting $\beta$-catenin to caveolae membrane domains," Journal of Biological Chemistry, vol. 275, no. 30, pp. 23368-23377, 2000.

[49] Y. Li, Z.-Q. Chen, and Y.-D. Li, "Effects of curcumin on the epithelial mesenchymal transition and TGF-beta/Smads signaling pathway in unilateral ureteral obstruction rats," Zhongguo Zhong Xi Yi Jie He Za Zhi, vol. 31, no. 9, pp. 1224-1228, 2011.

[50] R. Li, Y. Wang, Y. Liu et al., "Curcumin inhibits transforming growth factor- $\beta 1$-induced EMT via PPAR $\gamma$ pathway, not smad pathway in renal tubular epithelial cells," PLOS ONE, vol. 8, no. 3, Article ID e58848, 2013.

[51] X. Zhang, D. Liang, L. Guo et al., "Curcumin protects renal tubular epithelial cells from high glucose-induced epithelial-tomesenchymal transition through Nrf2-mediated upregulation of heme oxygenase-1," Molecular Medicine Reports, vol. 12, no. 1, pp. 1347-1355, 2015.
[52] Z. L. Li, L. Mo, G. Le, and Y. Shi, "Oxidized casein impairs antioxidant defense system and induces hepatic and renal injury in mice," Food and Chemical Toxicology, vol. 64, pp. 86-93, 2014.

[53] E. Tapia, V. Soto, K. M. Ortiz-Vega et al., "Curcumin induces Nrf2 nuclear translocation and prevents glomerular hypertension, hyperfiltration, oxidant stress, and the decrease in antioxidant enzymes in 5/6 nephrectomized rats," Oxidative Medicine and Cellular Longevity, vol. 2012, Article ID 269039, 2012.

[54] S. Boddupalli, J. R. Mein, S. Lakkanna, and D. R. James, "Induction of phase 2 antioxidant enzymes by broccoli sulforaphane: perspectives in maintaining the antioxidant activity of vitamins A, C, and E," Frontiers in Genetics, vol. 3, article 7, 2012.

[55] T. Nguyen, P. Nioi, and C. B. Pickett, "The Nrf2-antioxidant response element signaling pathway and its activation by oxidative stress," Journal of Biological Chemistry, vol. 284, no. 20, pp. 13291-13295, 2009.

[56] S.-Y. Lee, S. I. Kim, and M. E. Choi, "Therapeutic targets for treating fibrotic kidney diseases," Translational Research, vol. 165, no. 4, pp. 512-530, 2015.

[57] J. Gaedeke, N. A. Noble, and W. A. Border, "Curcumin blocks multiple sites of the TGF- $\beta$ signaling cascade in renal cells," Kidney International, vol. 66, no. 1, pp. 112-120, 2004.

[58] C.-C. Zeng, X. Liu, W.-W. Liu et al., "Protective effect of curcumin derivative B06 on kidney of type 2 diabetic rats," Chinese Journal of Applied Physiology, vol. 31, no. 1, pp. 38-42, 2015.

[59] C. Xin, S. Ren, B. Kleuser et al., "Sphingosine 1-phosphate crossactivates the Smad signaling cascade and mimics transforming growth factor- $\beta$-induced cell responses," The Journal of Biological Chemistry, vol. 279, no. 34, pp. 35255-35262, 2004.

[60] B. Ogretmen and Y. A. Hannun, "Biologically active sphingolipids in cancer pathogenesis and treatment," Nature Reviews Cancer, vol. 4, no. 8, pp. 604-616, 2004.

[61] X.-Y. Fu, D.-W. Zhang, Y.-D. Li et al., "Curcumin treatment suppresses CCR7 expression and the differentiation and migration of human circulating fibrocytes," Cellular Physiology and Biochemistry, vol. 35, no. 2, pp. 489-498, 2015.

[62] T. Xu, N.-S. Wang, L.-L. Fu, C.-Y. Ye, S.-Q. Yu, and C.-L. Mei, "Celecoxib inhibits growth of human autosomal dominant polycystic kidney cyst-lining epithelial cells through the VEGF/Raf/MAPK/ERK signaling pathway," Molecular Biology Reports, vol. 39, no. 7, pp. 7743-7753, 2012.

[63] S. Sun, X. Ning, Y. Zhai et al., "Egr-1 mediates chronic hypoxiainduced renal interstitial fibrosis via the PKC/ERK pathway," American Journal of Nephrology, vol. 39, no. 5, pp. 436-448, 2014.

[64] J. Ni, Y. Shen, Z. Wang et al., "P300-dependent STAT3 acetylation is necessary for angiotensin II-induced pro-fibrotic responses in renal tubular epithelial cells," Acta Pharmacologica Sinica, vol. 35, no. 9, pp. 1157-1166, 2014.

[65] Q. Li, X. Liu, and J. Wei, "Ageing related periostin expression increase from cardiac fibroblasts promotes cardiomyocytes senescent," Biochemical and Biophysical Research Communications, vol. 452, no. 3, pp. 497-502, 2014.

[66] Y. Wang, Y. Wang, M. Luo et al., "Novel curcumin analog C66 prevents diabetic nephropathy via JNK pathway with the involvement of p300/CBP-mediated histone acetylation," Biochimica et Biophysica Acta-Molecular Basis of Disease, vol. 1852, no. 1, pp. 34-46, 2015.

[67] M. Pang, J. Kothapally, H. Mao et al., "Inhibition of histone deacetylase activity attenuates renal fibroblast activation and 
interstitial fibrosis in obstructive nephropathy," American Journal of Physiology-Renal Physiology, vol. 297, no. 4, pp. F996F1005, 2009.

[68] Y. Okunuki, Y. Usui, H. Nakagawa et al., "Peroxisome proliferator-activated receptor- $\gamma$ agonist pioglitazone suppresses experimental autoimmune uveitis," Experimental Eye Research, vol. 116, pp. 291-297, 2013.

[69] H. Kusunoki, Y. Taniyama, H. Rakugi, and R. Morishita, "Cardiac and renal protective effects of irbesartan via peroxisome proliferator-activated receptor $\gamma$-hepatocyte growth factor pathway independent of angiotensin II Type la receptor blockade in mouse model of salt-sensitive hypertension," Journal of the American Heart Association, vol. 2, no. 2, Article ID e000103, 2013.

[70] E. L. Santos, K. de Picoli Souza, E. D. da Silva et al., "Long term treatment with ACE inhibitor enalapril decreases body weight gain and increases life span in rats," Biochemical Pharmacology, vol. 78, no. 8, pp. 951-958, 2009.

[71] S. Zhao, Y. Mugabo, J. Iglesias et al., " $\alpha / \beta$-Hydrolase domain6-accessible monoacylglycerol controls glucose-stimulated insulin secretion," Cell Metabolism, vol. 19, no. 6, pp. 993-1007, 2014. 

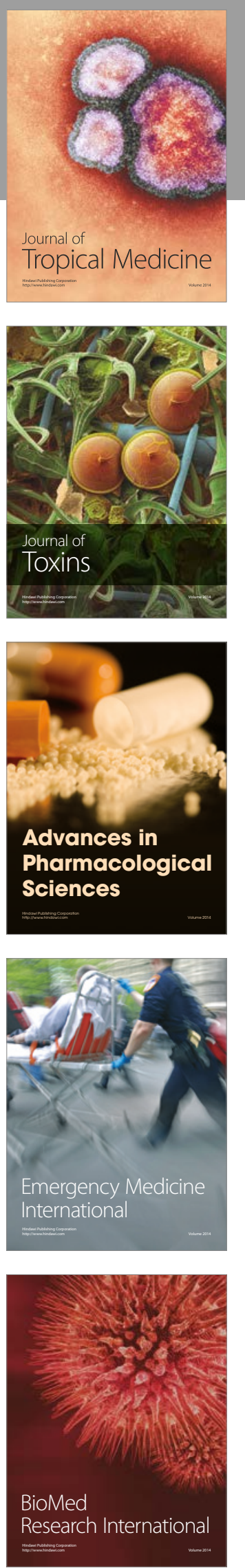
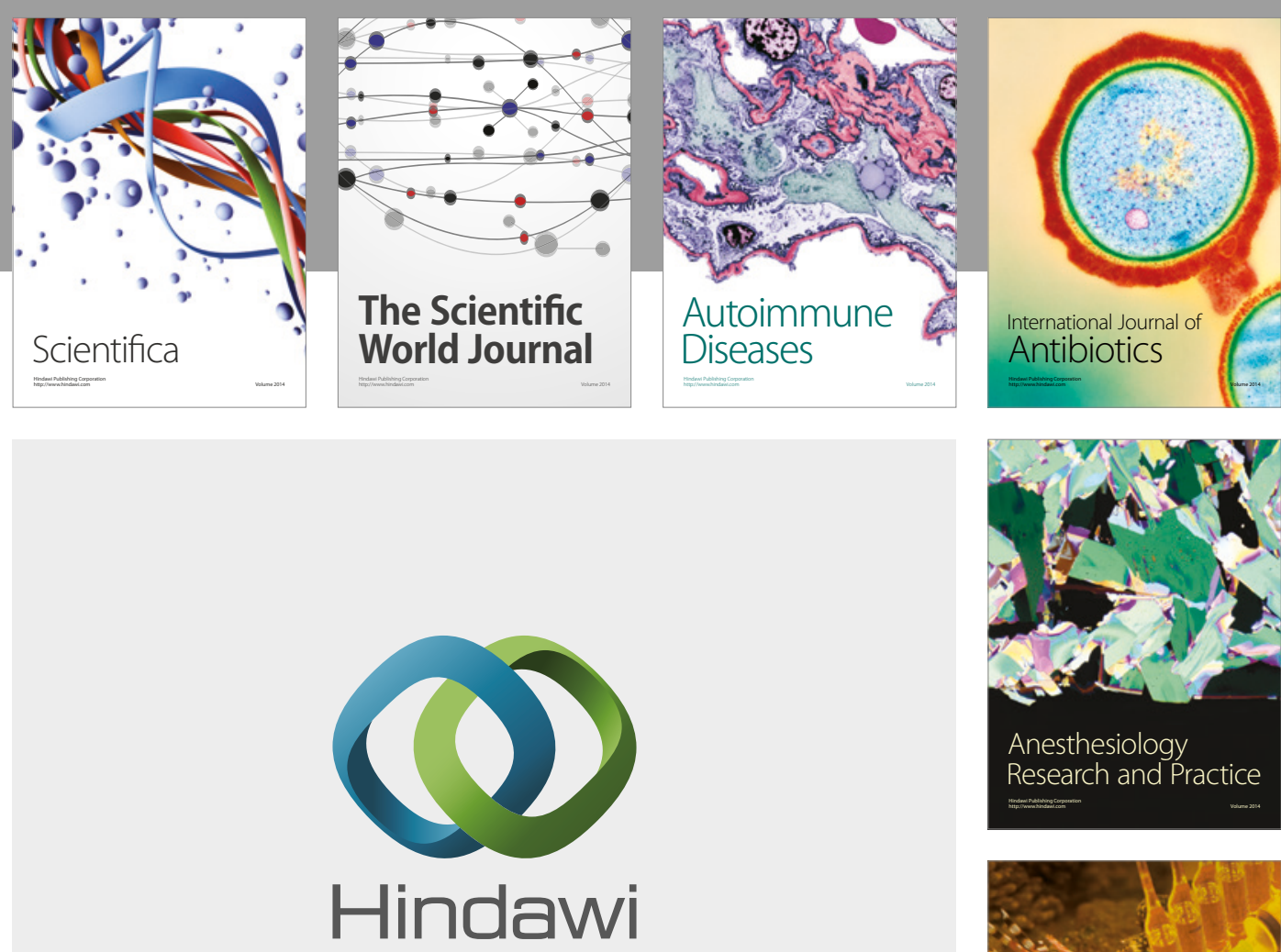

Submit your manuscripts at

https://www.hindawi.com
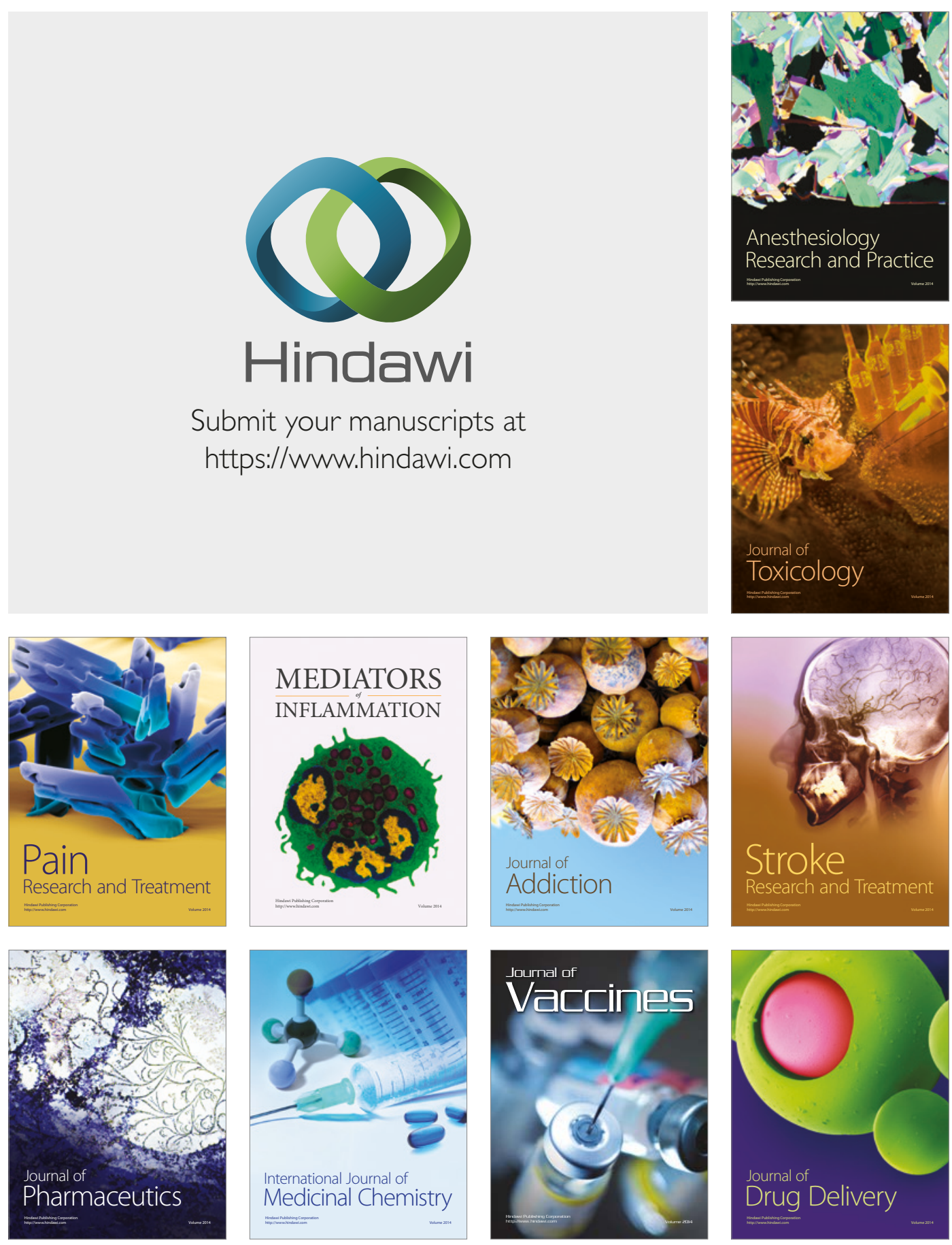